\title{
Ancient antagonism between CELF and RBFOX families tunes mRNA splicing outcomes
}

\author{
Matthew R. Gazzara, ${ }^{1,2}$ Michael J. Mallory, ${ }^{2}$ Renat Roytenberg, ${ }^{2}$ John P. Lindberg, ${ }^{2}$ \\ Anupama Jha, ${ }^{3}$ Kristen W. Lynch, ${ }^{1,2}$ and Yoseph Barash ${ }^{1,3}$ \\ ${ }^{1}$ Department of Genetics, ${ }^{2}$ Department of Biochemistry and Biophysics, Perelman School of Medicine, University of Pennsylvania, \\ Philadelphia, Pennsylvania 19104, USA; ${ }^{3}$ Department of Computer and Information Science, School of Engineering and Applied \\ Sciences, University of Pennsylvania, Philadelphia, Pennsylvania 19104, USA
}

\begin{abstract}
Over $95 \%$ of human multi-exon genes undergo alternative splicing, a process important in normal development and often dysregulated in disease. We sought to analyze the global splicing regulatory network of CELF2 in human T cells, a well-studied splicing regulator critical to $T$ cell development and function. By integrating high-throughput sequencing data for binding and splicing quantification with sequence features and probabilistic splicing code models, we find evidence of splicing antagonism between CELF2 and the RBFOX family of splicing factors. We validate this functional antagonism through knockdown and overexpression experiments in human cells and find CELF2 represses RBFOX2 mRNA and protein levels. Because both families of proteins have been implicated in the development and maintenance of neuronal, muscle, and heart tissues, we analyzed publicly available data in these systems. Our analysis suggests global, antagonistic coregulation of splicing by the CELF and RBFOX proteins in mouse muscle and heart in several physiologically relevant targets, including proteins involved in calcium signaling and members of the MEF2 family of transcription factors. Importantly, a number of these coregulated events are aberrantly spliced in mouse models and human patients with diseases that affect these tissues, including heart failure, diabetes, or myotonic dystrophy. Finally, analysis of exons regulated by ancient CELF family homologs in chicken, Drosophila, and Caenorhabditis elegans suggests this antagonism is conserved throughout evolution.
\end{abstract}

[Supplemental material is available for this article.]

Alternative splicing of pre-mRNAs is required for proper protein expression and cellular function across all metazoans (Kalsotra and Cooper 2011). Alternative splicing is typically controlled by binding of proteins along a nascent transcript (Fu and Ares 2014). These RNA binding proteins (RBPs) in turn regulate the interaction of the splicing machinery with substrate, resulting in inclusion or skipping of specific exons in the final mRNA. Importantly, pre-mRNA transcripts are often bound by multiple proteins that cooperate or compete in their regulation of the splicing machinery. Thus, the final splicing pattern of a gene in any given cell is determined by the combinatorial activity of the repertoire of RNA binding proteins associated with its pre-mRNA (Fu and Ares 2014). It follows that understanding the relative expression and activity of RNA binding proteins is essential to determining how gene expression is controlled.

Two RBPs that have been particularly linked to alternative splicing in many developmental and differentiation processes are CELF2 and RBFOX2. CELF2 is one of the six members of the human family of CUGBP, ELAV-like family (CELF) proteins, while RBFOX2 is a member of the RBFOX family of RBPs, which has three paralogs in humans. Both families of RBPs are strongly conserved throughout metazoa and have been well-documented to regulate tissue- and developmental-specific splicing from Caenorhabditis elegans to humans. In humans, the different members of the CELF and RBFOX families exhibit distinct tissue distribution and their expression is often highly regulated in a developmental-de-

Corresponding authors: yosephb@upenn.edu, klync@mail.med. upenn.edu

Article published online before print. Article, supplemental material, and publication date are at http://www.genome.org/cgi/doi/10.1101/gr.220517.117. pendent manner (Dasgupta and Ladd 2011; Conboy 2016). For example, CELF2 is highly expressed in the fetal heart but shows a marked reduction in expression during post-natal development, which correlates with widespread changes in alternative splicing (Ladd et al. 2005; Kalsotra et al. 2008, 2010). Altered expression of CELF2 in neuronal and muscle cells has also been shown to correlate with splicing changes (Charlet et al. 2002; Dasgupta and Ladd 2011; Wang et al. 2015). Although these studies have not demonstrated a causal role of CELF2 in the observed splicing changes, CELF2 expression does increase in developing thymocytes and activated $\mathrm{T}$ cells and regulates splicing to promote $\mathrm{T}$ cell receptor expression and signaling (Mallory et al. 2011; Martinez et al. 2015). Similarly, proper expression of RBFOX2 in brain and muscle of several species is required for appropriate splicing and tissue function (Kuroyanagi et al. 2007; Gallagher et al. 2011; Singh et al. 2014). Interestingly, despite the overlap in expression and function of CELF2 and RBFOX2 in many tissues and across species, physical or functional interplay between these proteins has not been well studied.

Recently, we have shown that CELF2 expression increases during thymic development and upon activation of mature T cells (Mallory et al. 2011, 2015). We have also identified CELF2-dependent alternative splicing of $\sim 100$ genes in immature, mature, and cultured $\mathrm{T}$ cells and have mapped the transcriptome-wide association of CELF2 with pre-mRNA in cultured Jurkat T cells (Mallory et al. 2015; Martinez et al. 2015; Ajith et al. 2016). These studies

(C) 2017 Gazzara et al. This article is distributed exclusively by Cold Spring Harbor Laboratory Press for the first six months after the full-issue publication date (see http://genome.cshlp.org/site/misc/terms.xhtml). After six months, it is available under a Creative Commons License (Attribution-NonCommercial 4.0 International), as described at http://creativecommons.org/licenses/ by-nc/4.0/. 
revealed that CELF2 functions generally in a position-dependent manner, in which binding of CELF2 upstream of a variable exon results in exon repression, while binding of CELF2 downstream from a variable exon induces its inclusion in the final mRNA (Ajith et al. 2016). Interestingly, our initial analysis of CELF2 binding compared to function also indicated that many of the $\sim 100$ genes for which we observed differential splicing in CELF2-depleted cells lacked observable binding of CELF2 around the regulated exon (Ajith et al. 2016). Here, we investigate the mechanistic underpinning of these exons that are regulated, but not bound, by CELF2.

\section{Results}

CELF2 regulates an extensive program of splicing in T cells both directly and indirectly

In previous studies, we used a targeted RNA-seq approach to identify $\sim 100$ cassette exons that exhibit altered splicing upon knockdown of CELF2 expression in Jurkat T cells (Mallory et al. 2015; Martinez et al. 2015; Ajith et al. 2016). As this pilot study interrogated only $\sim 5000$ exons, we sought to gain a more complete view of the consequence of CELF2 expression on the transcriptome in T cells using RNA-seq of total poly(A)-selected RNA from Jurkat cells. We have previously shown that CELF2 expression increases upon stimulation of Jurkat cells with the phorbol ester PMA (Mallory et al. 2015; Martinez et al. 2015). Therefore, we carried out RNA-seq of mRNA from wild-type and CELF2-depleted Jurkat cells grown under either unstimulated or PMA-stimulated conditions. Depletion of CELF2 was done by doxycycline-inducible expression of a shRNA targeting CELF2, as we have described previously (Mallory et al. 2015; Martinez et al. 2015; Ajith et al. 2016).

Using the splicing-quantification algorithm MAJIQ (Vaquero-Garcia et al. 2016), we identified 901 significant changes in splicing (local splicing variations or LSVs) induced upon CELF2 depletion in unstimulated cells and $~ 1700$ significant CELF2-dependent LSVs in stimulated cells (Fig. 1A). The larger number of CELF2-dependent LSVs in stimulated cells is consistent with the increased expression of CELF2 in these cells compared to unstimulated conditions. Despite the difference in number of CELF2-dependent LSVs in these two cell conditions, there is a significant overlap in the identity of CELF2-regulated LSVs in unstimulated and stimulated cells (Fig. 1A). Moreover, the extent of CELF2 regulation of individual LSVs in these two conditions is also highly similar (Fig. 1B), as we have observed previously (Ajith et al. 2016). Therefore, we conclude that CELF2 has a widespread and consistent impact on alternative splicing in both unstimulated and stimulated $\mathrm{T}$ cells. For subsequent analysis, we have thus merged together the high-confidence LSVs from unstimulated and stimulated cells (i.e., those with a probability of $>95 \%$ that the difference in inclusion (delta PSI or $\Delta \Psi$ ) is $>20 \%$ ) to identify sets of cassette exons for which inclusion is increased (CELF2-repressed) or decreased (CELF2-enhanced) upon depletion of
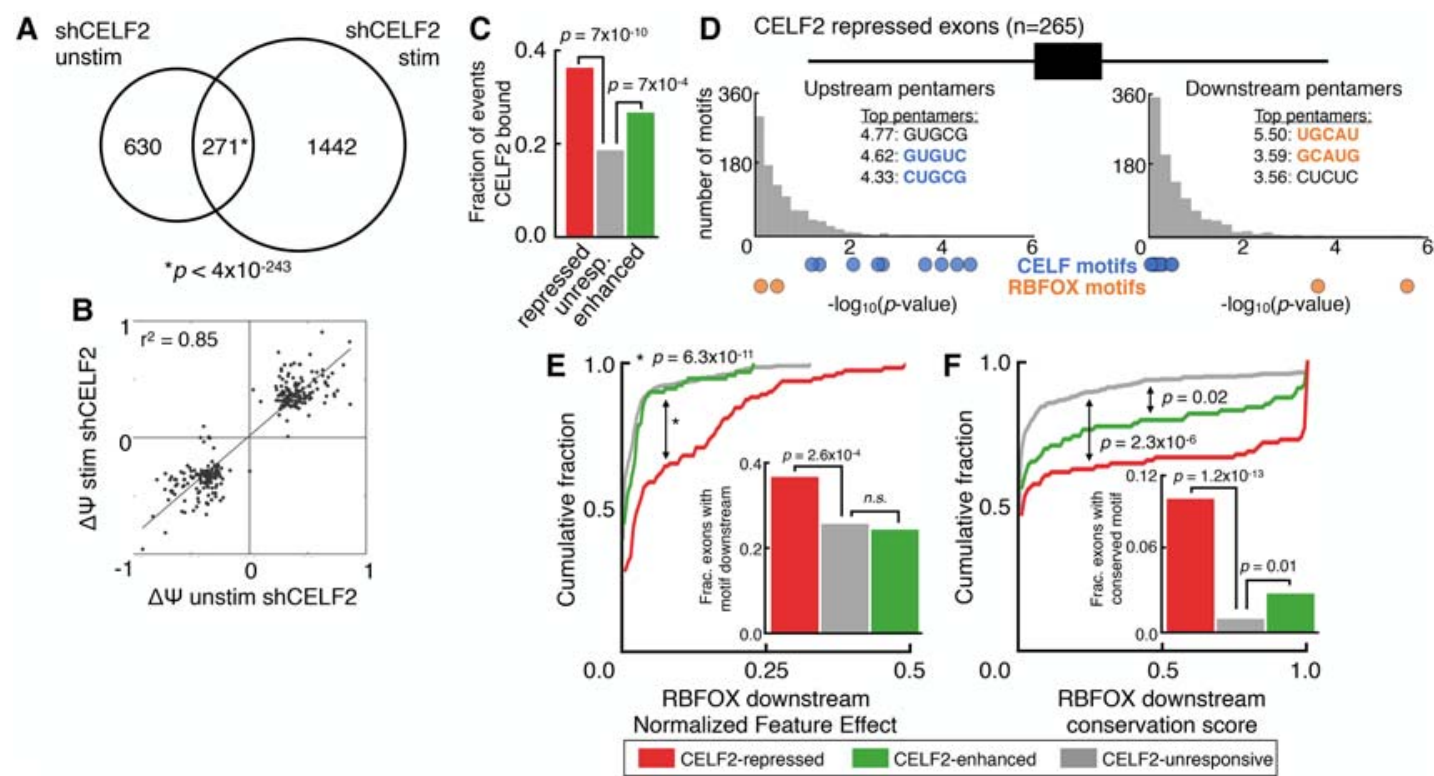

Figure 1. RNA-seq reveals global CELF2 regulation in T cells and sequence analysis suggests coregulation by the RBFOX family. $(A)$ Venn diagram of the overlap of unique, high-confidence LSVs (Vaquero-Garcia et al. 2016) that showed significantly altered splicing $(|\Delta \Psi| \geq 20 \%$ with probability $\geq 95 \%)$ upon depletion of CELF2 by shRNA in unstimulated (left), stimulated (right), or both (middle) JSL1 Jurkat T cells (Fisher's exact (FE) test, two-tailed $P<7 \times 10^{-192}$ ). (B) Scatterplot comparing $\mathrm{E}(\Delta \Psi)$ values for the most changing junction from the 271 unique, coregulated LSVs upon CELF2 depletion in unstimulated $(X)$ and stimulated $(Y)$ Jurkat T cells. (C) Fraction of high-confidence cassette exons derived from the union of events in panel $A$, containing splice site proximal CELF2 CLIP-seq peaks that were repressed by CELF2 (red, $n=265$ ), enhanced by CELF2 (green, $n=371$ ), or unresponsive (gray, $n=1668$ ) (FE test, twotailed). (D) Distribution of enrichment significance (- $\log _{10}[P$-value], hypergeometric test comparing regulated to unresponsive sets) for the occurrence of all pentamers upstream (left) or downstream (right) of CELF2-repressed exons. Top three pentamers for each are shown in the inset and motifs known to be bound by the CELF family or RBFOX family are highlighted in blue or orange, respectively. See Supplemental Table S2 for all values. (E) Cumulative distribution function (CDF) of the AVISPA predicted impact on splicing (normalized feature effect, NFE) (Barash et al. 2013) for downstream [U]GCAUG motifs for the subsets of events that contained it (Kolmogorov-Smirnov two-sample test). Fraction of each set with a downstream [U]GCAUG is given in inset with colors corresponding to the key (FE test, two-tailed). ( $F$ ) CDF showing the average conservation (phastCons 46way, placental mammals) for the core GCAUG motif for the subsets of events that contained it downstream of the alternative exon (Kolmogorov-Smirnov [KS] two-sample test). Fraction of each set with a highly conserved downstream GCAUG (see Methods) is given in inset with colors corresponding to the key (FE test, two-tailed). 
CELF2, in addition to exons that were unresponsive to CELF2 depletion (CELF2-unresponsive). This resulted in 265 CELF2-repressed, 371 CELF2-enhanced, and 1668 CELF2-unresponsive exons (Supplemental Table S1; see Methods). We note that the final number of CELF2-regulated events defined this way (265+ $371=636$ ) is less than those in Figure $1 \mathrm{~A}$ due to restricting the list to cassette exons (to facilitate subsequent analysis) and to the fact that multiple LSVs can report on the same cassette exon (Vaquero-Garcia et al. 2016).

To determine the extent to which binding of CELF2 correlates with splicing, we overlaid our newly identified functional targets of CELF2 with the CELF2 binding sites we previously defined in Jurkat cells (Ajith et al. 2016). Consistent with our prior analysis of the more limited set of CELF2-regulated exons (Ajith et al. 2016), we find that CELF2 binds preferentially around cassette exons that it regulates (Fig. 1C), suggesting a direct role of CELF2 in regulation of many of the CELF2-responsive exons. However, for two-thirds of CELF2-responsive exons, we find no evidence for CELF2 binding in locations that are commonly associated with splicing regulation (within the regulated exon, flanking constitutive exons, or 300 nucleotides [nt] of flanking intron) (Fig. 1C). While we cannot rule out that in some of these instances CELF2 functions from a distance of $>300 \mathrm{nt}$, or that the CLIP data are not saturating and have missed some binding sites, these results strongly suggest that CELF2 regulates a sizeable number of exons by an indirect mechanism.

\section{RBFOX motifs and binding are enriched downstream of CELF2-repressed exons}

To investigate potential indirect mechanisms for CELF2 regulation of splicing, we looked for enriched pentamers within $300 \mathrm{nt}$ upstream or downstream of CELF2-repressed or enhanced exons using a hypergeometric test (Fig. 1D; Supplemental Table S2). Consistent with previous findings where CELF2 was shown to repress exon inclusion when bound upstream of cassette exons (Han and Cooper 2005; Dembowski and Grabowski 2009; Ajith et al. 2016), we see specific enrichment of UG-rich pentamers upstream of CELF2-repressed exons (Fig. 1D, left, blue circles). Strikingly, in contrast, we found that the top two pentamers (UGCAU and GCAUG) enriched downstream of CELF2-repressed exons (Fig. 1D, right, orange circles) are perfect matches to the high-affinity binding site of the RBFOX family, [U]GCAUG (Ponthier et al. 2006). Notably, this enrichment of RBFOX pentamers was specific to the downstream intron, where RBFOX typically functions as a potent enhancer of exon inclusion (Yeo et al. 2009). While several other pentamers are also enriched around both CELF2-enhanced and -repressed exons (Supplemental Table S2), we focused further investigation on the RBFOX motif for two reasons. First, the enrichment of the RBFOX motif downstream of CELF2-repressed exons is among the most significant, even more so than that of the UG-rich CELF2 binding sites upstream of these same exons (Fig. 1D). Secondly, the CELF and RBFOX families have both been implicated in the development and maintenance of normal function of heart, muscle, and brain (Zhang et al. 2002; Kalsotra et al. 2008; Gehman et al. 2012; Singh et al. 2014) but have yet to be directly studied in connection to one another.

We first utilized the AVISPA tool (Barash et al. 2013) to predict the relevance of the RBFOX motifs in determining splicing outcome. Consistent with the pentamer enrichment described above, AVISPA found the RBFOX motif occurred significantly more often downstream of CELF2-repressed exons (36.6\%) compared to unresponsive exons $\left(25.6 \%\right.$, Fisher's exact two-tailed test, $\left.P<3 \times 10^{-4}\right)$ or CELF2-enhanced exons $\left(24.1 \%, P<1 \times 10^{-3}\right)$ (Fig. $1 \mathrm{E}$ inset). Moreover, the presence of the RBFOX motif downstream from CELF2-repressed exons is predicted by AVISPA to have significantly more impact on splicing (higher normalized feature effect) (Barash et al. 2013; see Supplemental Methods) when compared to either unresponsive (Kolmogorov-Smirnov two-sample test, $P<$ $\left.6.3 \times 10^{-11}\right)$ or CELF2-enhanced exons $\left(P<2 \times 10^{-4}\right)$ (Fig. 1E). The RBFOX motifs downstream of the CELF2-repressed exons are also more highly conserved than those around CELF2-unresponsive exons (Fig. 1F), which is an additional hallmark of functional relevance (Lambert et al. 2014; Taliaferro et al. 2016).

Finally, we utilized publicly available eCLIP peaks for RBFOX2 from a variety of cell types including HEK293, human embryonic stem cells (H1ES), HepG2, and K562 cells (Conway et al. 2016; Sundararaman et al. 2016; Van Nostrand et al. 2016) to see if there was evidence of in vivo binding of RBFOX2 in regions proximal to CELF2-regulated cassette exons. In line with the sequence enrichment data, we find enrichment of RBFOX2 eCLIP binding sites around CELF2-repressed exons, compared to unresponsive exons, in all four cell types examined (from $~ 14 \%$ to $25 \%$ ) (Fig. 2A, top). Although there was significant enrichment of RBFOX2 eCLIP peaks in most regions proximal to and within CELF2-repressed exons, the most striking enrichment in all cell types was for binding downstream of the cassette exon (Fig. 2A, bottom). This enrichment of RBFOX2 binding downstream of CELF2-repressed exons is unique compared to other RBPs analyzed by eCLIP and iCLIP experiments from ENCODE, including CELF2 (Fig. 2B), and is also observed when the RBFOX2 eCLIP peaks are mapped at a nucleotide level around CELF2-repressed exons (Fig. 2C). Notably, this enrichment of RBFOX2 binding downstream of the $5^{\prime}$ ss, a region that has been previously identified as a location from which RBFOX2 acts as a strong enhancer of exon inclusion (Yeo et al. 2009), predicts that RBFOX proteins likely enhance the inclusion of exons deemed to be CELF2-repressed.

\section{CELF2 and RBFOX2 antagonize splicing patterns in T cells}

To directly test the functional relevance of RBFOX binding to CELF2-responsive exons, we analyzed the relative impact of CELF2 and RBFOX depletion on the splicing of these exons. Jurkat T cells express no detectable RBFOX1 or RBFOX 3 mRNA (data not shown) but do express moderate levels of RBFOX2 mRNA and protein (see below), consistent with restricted expression of RBFOX1 (muscle, heart, and brain) and RBFOX3 (brain) but more widespread expression of RBFOX2 (Conboy 2016). Therefore, we analyzed the splicing of CELF2-repressed exons in Jurkat cells depleted of CELF2, RBFOX2, or both proteins. Of the 265 high-confidence CELF2-repressed exons, 127 have evidence for RBFOX family binding in the downstream intron (eCLIP peak and/or [U]GCAUG motif). For 37 of these, we also detect CELF2 binding upstream (14) (Fig. 3A), downstream (5) (Fig. 3C), or on both sides (18) (Fig. 3B) of the regulated exon, while the remaining 90 have no CELF2 CLIP peaks in the vicinity of the CELF2-responsive exon (Fig. 3D). The very small number of CELF2-repressed exons with CELF2 binding only downstream is consistent with our earlier finding that binding of CELF2 downstream from an exon correlates mostly with exon inclusion, while binding of CELF2 upstream correlates with exon skipping (Ajith et al. 2016). Moreover, the proportion of RBFOX bound events that

\section{Genome Research}

www.genome.org 
A

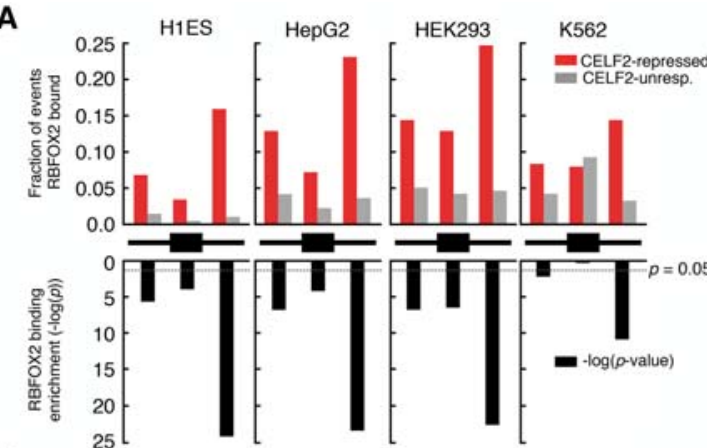

B

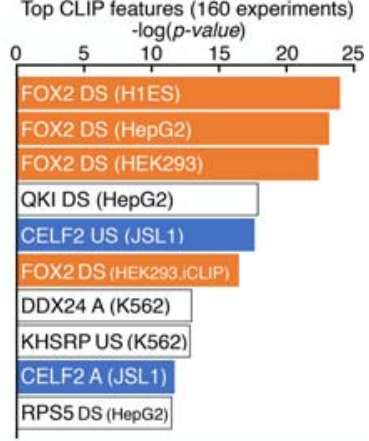

C
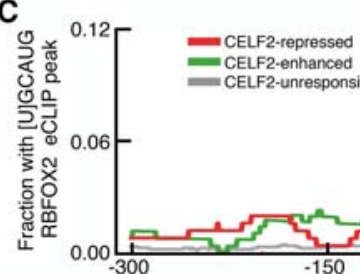
- CELF2-enhanced - CELF2-unresponsive (1)$$
-150 \quad \text { Distance from alternative exon (nt) }
$$

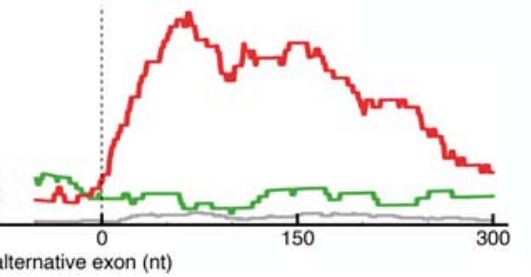

$$
\text { Distance from alternative exon ( } n t)
$$

Figure 2. CELF2-repressed exons are highly enriched for downstream in vivo binding of RBFOX2. (A) Fraction of exons that were repressed by CELF2 (red) or unresponsive (gray) that contained an RBFOX2 eCLIP peak from indicated cell type in regions proximal to the alternative exon (black diagram: within $300 \mathrm{nt}$ upstream, within the exon, or within $300 \mathrm{nt}$ downstream) (top) and $-\log _{10}(P$-value) of enrichment of RBFOX2 eCLIP peak occurrence in the CELF2-repressed versus unresponsive set (bottom, black bars, Fisher's exact test, two-tailed $P$ ). (B) Top 10 enriched CLIP features found when comparing CLIP peak occurrence from 160 experiments (ENCODE eCLIP and iCLIP) (Ajith et al. 2016) between CELF2-repressed and unresponsive exons. Condition in which each CLIP experiment was carried out is indicated in parentheses. CLIP experiments for RBFOX2 or CELF2 are indicated in orange or blue, respectively. (DS) Bound downstream of alternative exon, (US) bound upstream of alternative exon, (A) bound within alternative exon. (C) RNA maps showing the per-nucleotide frequency of [U]GCAUG containing RBFOX2 eCLIP peak occurrences within 300 nt of alternative exons that are repressed by CELF2 (red), enhanced by CELF2 (green), or were unresponsive to depletion (gray).

exhibit CELF2 binding is consistent with the percent of total CELF2-repressed exons that have evidence for CELF2 binding (Fig. 1C).

Notably, regardless of the presence or absence of detectable CELF2 or RBFOX2 binding sites, all 20 of the 265 high-confidence CELF2-repressed exons tested show increased inclusion upon depletion of CELF2 by shRNA (Fig. 3A-E; Supplemental Figs. S1, S2), validating our identification of CELF2-repressed exons from the RNA-seq data. Interestingly, depletion of RBFOX2 alone does have an opposite impact from CELF2 on these exons, consistent with functional antagonism; however, this impact of RBFOX2 depletion is only notable for a handful of exons compared to wild-type cells (e.g., MYL6, MYO9B). In contrast, in the codepletion of RBFOX2 and CELF2, we observe a striking antagonistic relationship that can be grouped into two patterns. For those exons with binding of CELF2 upstream, where we predict direct repression by CELF2, codepletion of RBFOX2 has a notable but modest impact on splicing relative to CELF2 alone (Fig. 3A, blue vs. dark gray), and depletion of CELF2 has a significant impact on exon inclusion even in the absence of RBFOX2 (Fig. 3A, orange vs. dark gray). On the other hand, for those exons around which we detect no binding of CELF2, codepletion of RBFOX2 completely abrogates the effect of CELF2 depletion (Fig. 3D, light gray vs. dark gray or blue), indicating that the impact of CELF2 depletion is primarily mediated indirectly via RBFOX2 (see below). Exons bound on either side by CELF2 exhibit a pattern most similar to the exons with no CELF2 binding, suggesting that perhaps in this case the enhancing and repressing activities of CELF2 cancel each other out and splicing is ultimately directed by RBFOX2 (Fig. 3B). In contrast, exons that have binding sites for both CELF2 and RBFOX2 downstream show a mixed response of codepletion, with most events resembling RBFOX2-driven splicing (NF2, MACF1, and FLNB) (Fig. 3C), consistent with CELF2 not typically repressing from a downstream location, while other events show a more complicated response to codepletion (Fig. 3B, SLMAP). Importantly, we observe no impact of RBFOX2 depletion on CELF2-repressed exons that lack evidence for RBFOX2 binding (Fig. 3E).

In sum, we conclude that for CELF2bound and -repressed targets, CELF2 is the primary driver of splicing, but many of these genes are also bound and enhanced by RBFOX2, which partly counters the repressive activity of CELF2. Critically, we also demonstrate that a large percentage (at least 90 of the 265 or 34\%) of CELF2-repressed exons are unlikely to be direct targets of CELF2 but rather are regulated primarily through RBFOX2. Consistent with this interpretation, overexpression of RBFOX2 phenocopies depletion of CELF2 for all four of the CELF2-repressed genes tested for which we have no evidence of CELF2 binding (cf. Fig. 3, F and D; Supplemental Figs S2E, S3), while overexpression of RBFOX2 has a more minimal effect on genes in the other subgroups (Fig. 3A-C,E; Supplemental Fig. S2E).

\section{CELF2 represses RBFOX2 mRNA and protein levels in T cells}

One mechanism that could explain the remarkable impact of RBFOX2 on indirect targets of CELF2 regulation would be if depletion of CELF2 alters the expression of RBFOX2 in Jurkat cells. Consistent with this model, analysis of RBFOX2 mRNA expression upon CELF2 depletion by RNA-seq revealed up-regulation in RBFOX2 mRNA levels of greater than 2.5-fold in both unstimulated and stimulated Jurkat cells upon depletion of CELF2 (adjusted $P<2.3 \times 10^{-11}$ ) (Fig. 4A,B). Western blotting confirmed that this up-regulation of $R B F O X 2$ is also manifest at the protein level upon CELF2 depletion (Fig. 4C). This increase in RBFOX2 protein upon CELF2 depletion occurs both in unstimulated cells, in which CELF2 expression is low, and in stimulated cells, in which CELF2 expression is higher (Fig. 4C, cf. lanes 1 and 2 for unstimulated [left] and stimulated [right] cells). Notably, CELF2 expression is sufficient to regulate RBFOX2, independent of cell type, as overexpression of FLAG-tagged CELF2 reduced RBFOX2 expression both in Jurkat cells as well as HEK293 cells. However, the antagonistic relationship between CELF2 and RBFOX2 expression is unidirectional, at least in Jurkat cells, as neither depletion nor overexpression of RBFOX2 caused an appreciable change in CELF2 protein (Fig. 4C, cf. lanes 1 and 3 for both; Supplemental Fig. S3).

RBFOX2 is known to contain several alternative splicing events, including exon skipping of part of the single RRM which 


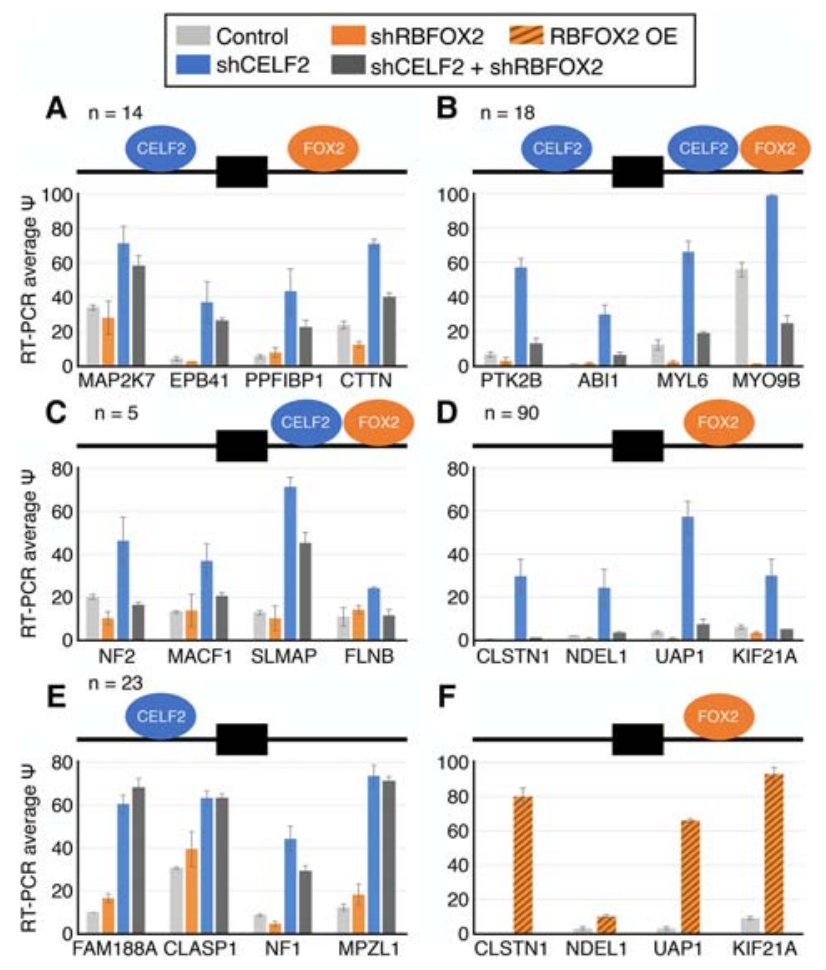

Figure 3. Reciprocal regulation of CELF2-repressed exons by RBFOX2 in T cells. (A-E) Quantification of splicing by RT-PCR (see Supplemental Fig. S1 for gels) of MAJIQ-identified CELF2-repressed exons that contain proximal intronic binding of CELF2 (CLIP peak) and/or RBFOX2 (CLIP peak and/or GCAUG) in wild-type Jurkat T cells (light gray), or cells depleted of RBFOX2 (orange), CELF2 (blue), or both proteins (dark gray) by shRNA. Efficient depletion of proteins was confirmed via Western blot (see Fig. 4). Mean and standard deviation (error bars) are shown $(n \geq 3)$. Diagrams above bar charts indicate binding locations of CELF2 and/or RBFOX2 upstream or downstream of the alternative exon. $n$ represents the number of cassette exons exhibiting that binding pattern. Data shown are from stimulated cells; data for unstimulated cells are provided in Supplemental Figure S2. (F) Quantification of splicing by RT-PCR in wild-type Jurkat T cells (light gray) or those overexpressing RBFOX 2 CDNA (orange stripe). Protein expression and RT-PCR gels are in Supplemental Figure S3.

creates a dominant-negative version of the protein and an event in the C-terminal domain that can alter localization (Underwood et al. 2005; Damianov and Black 2010). However, analysis of RNA-seq data revealed no significant change in either RBFOX2 splicing event upon CELF2 depletion (Supplemental Fig. S4A). On the other hand, we do find a marked increase in the stability of RBFOX2 mRNA in CELF2-depleted versus wild-type Jurkat cells (Supplemental Fig. $\mathrm{S} 4 \mathrm{~B})$. This CELF2-dependent regulation of RBFOX2 stability is unlikely to be through regulation of nonsense-mediated mRNA decay, as we observe no change in RBFOX2 expression upon depletion of UPF1 (Supplemental Fig. S4C). We also observe little evidence of CELF2 binding to the 3' UTR of RBFOX2 (Supplemental Fig. S4D), suggesting that CELF2 may regulate the stability of RBFOX2 through an indirect mechanism.

\section{CELF/ RBFOX antagonism is conserved in mouse heart and muscle}

Given the striking functional antagonism between CELF2 and RBFOX2 in Jurkat T cells, we wished to explore if this antagonism was also evident in heart and muscle-systems in which both the CELF and RBFOX families of splicing factors are well studied and functionally critical. Previous work has shown that CELF1 and
CELF2 promote embryonic splicing patterns in murine muscle and heart that are reversed when both proteins are dramatically down-regulated post-natally (Ladd et al. 2005; Kalsotra et al. 2008). On the other hand, RBFOX1 and RBFOX2 have been implicated as key in promoting splicing changes during myogenesis and across heart and muscle development (Gallagher et al. 2011; Gehman et al. 2012; Singh et al. 2014; Pedrotti et al. 2015), yet these two protein families have not been studied in relation to one another in these contexts.

We identified CELF-regulated cassette exons using publicly available RNA-seq data from adult mouse hearts that ectopically expressed CELF1 or CELF2 (Giudice et al. 2014; Wang et al. 2015) and analyzed these cassette exons for enriched sequences and CLIP-seq features as done in the analysis of the human $\mathrm{T}$ cell data above. In line with the results from T cells, we found enriched occurrences of highly conserved [U]GCAUG motifs (see Methods) and RBFOX2 CLIP-seq peaks downstream of $12 \%$ $18 \%$ of CELF-repressed exons (Supplemental Fig. S5). The most enriched CLIP-seq feature was for downstream binding of RBFOX2 from mouse cardiomyocytes (Fig. 5A, left). RNA maps for the per nucleotide occurrence of these RBFOX2 CLIP peaks over CELF2-repressed exons showed clear enrichment downstream in a similar region as we observed for T cells (Fig. 5B).

Interestingly, RBFOX2-enhanced cassette exons from myotube data showed strong enrichment of CELF1 CLIP peaks proximal to the regulated exons, suggesting that $\mathrm{CELF} / \mathrm{RBFOX}$ antagonism may be a defining characteristic of not only CELF-regulated exons but also make up a significant proportion of RBFOX-regulated exons. To further investigate this possibility, we looked for shared splicing changes identified by a targeted sequencing approach (RASL-seq) (Li et al. 2012) from hearts of Rbfox2 knockout mice (Wei et al. 2015) and compared it to those identified by RNA-seq data from CELF1 or CELF2 overexpression in adult hearts (see Methods). Consistent with CELF/RBFOX antagonism being important for both CELF and RBFOX regulated exons in heart, there was a significant overlap in splicing changes (Fisher's exact two-tailed test, $P<2.6 \times 10^{-10}$ ), with $22 \%-30 \%$ of events in either set being coregulated (Fig. 5C). As a positive control, we repeated this analysis for hearts depleted of other splicing factors, including other UGrich binding proteins HNRNPU and RBM24, and found the CELF family to coregulate the largest fraction of RBFOX2 events (Fig. $5 \mathrm{D}$, top) with the most significant overlap of all RBPs tested (Fig. $5 \mathrm{D}$, bottom).

Notably, a majority of these coregulated events showed splicing changes in the same direction upon overexpression of CELF1 or CELF2 in the adult heart compared to depletion of RBFOX2 (Fig. 5D, gray). To assess if this apparent antagonism is true globally in the murine heart/muscle context, we compared all coregulated splicing changes quantified from RNA-seq. We again observe strong positive correlations between contexts in which CELF proteins were overexpressed compared to RBFOX2 depletion in myotubes (Pearson's $r \geq 0.7$ ) (Fig. 5E), suggesting that induced changes are not only in the same direction but also of similar magnitude. Taken together, these results suggest CELF/RBFOX antagonism is conserved in murine muscle and heart and broadly contributes to the regulatory programs controlled by each family.

\section{CELF and RBFOX cobind regulated exons in heart development and disease}

Given the evidence of conserved CELF and RBFOX family antagonism in mouse heart and muscle, we wished to examine if this

\section{Genome Research}

www.genome.org 
A

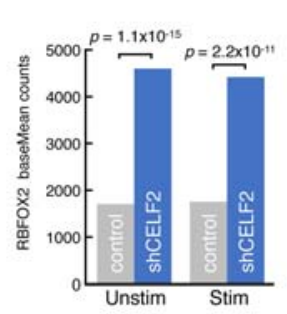

B

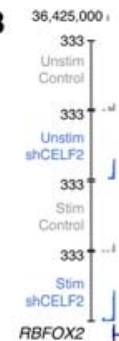

36.335,000
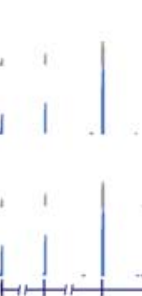

D
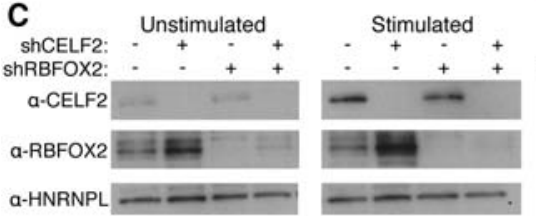
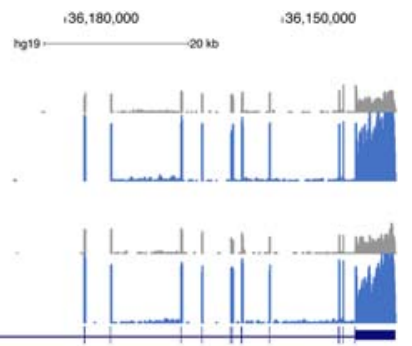

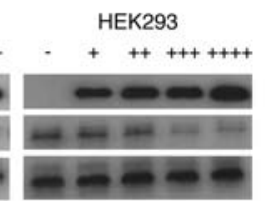

Figure 4. CELF2 represses RBFOX2 mRNA and protein levels in T cells. (A) RBFOX2 mRNA expression in unstimulated and stimulated JSL1 T cells (gray) compared to CELF2 depletion (blue) as determined by DESeq. Significant increases in RBFOX2 mRNA levels upon CELF2 depletion was determined by DESeq (fold-change $>2.5$ and adjusted $P<2.3 \times 10^{-11}$ ). (B) UCSC Genome Browser snapshot of RNA-seq reads over the RBFOX2 locus in unstimulated (top) or stimulated (bottom) control (gray) versus CELF2 depletion (blue) JSL1 cells. (C) Western blot monitoring protein levels of CELF2 and RBFOX2 in unstimulated (left) or stimulated (right) JSL1 cells depleted for CELF2 (lane 2), RBFOX2 (lane 3), or both (lane 4). An antibody against HNRNPL was used as a loading control. (D) Western blot monitoring FLAG-CELF2 and RBFOX2 expression in unstimulated JLS1 cells (left) or HEK293 cells (right) that expressed increasing amounts of FLAG-CELF2 construct. An antibody against HNRNPL was used as a loading control.

relationship influenced splicing changes across development or in disease where both families, individually, have been implicated. In line with previous results (Kalsotra et al. 2008; Gallagher et al. 2011), both CELF family overexpression in adult tissues or RBFOX2 depletion largely reversed changes that occurred from embryonic to adult heart development (Fig. 5E). RBFOX2 has been shown to be dysregulated in the hearts of diabetic patients and a mouse model of Type I diabetes (T1D), where it forms a dominant negative version of the protein, leading to a number of splicing changes (Nutter et al. 2016). CELF1 has also been shown to be up-regulated in diabetic hearts via PKC signaling (KuyumcuMartinez et al. 2007; Verma et al. 2013), but the global consequences of this have not been examined. By analyzing public data, we observe not only the previous finding that RBFOX2 depletion largely mirrors changes induced in a mouse model of T1D $(r=$ 0.44) (Fig. 5E) but, importantly, demonstrate that overexpression of CELF1 or CELF2 also mirrors T1D heart dysregulation, in all cases more strongly than RBFOX2 depletion $(0.45 \leq r \leq 0.72)$ (Fig. $5 \mathrm{E})$.

A large fraction of cassette exons that are regulated across development $(n=678)$ or dysregulated in T1D hearts $(n=1020)$ also have evidence for splice site-proximal binding in the heart by CELF1 (34.8\% of developmental, $28.8 \%$ of T1D) or RBFOX2 ( $28.8 \%$ of developmental, $18.7 \%$ of T1D) (Fig. 5F). Importantly, there is a significant overlap between the events in both contexts $\left(P<1.4 \times 10^{-16}\right)$ such that, in most cases, a majority of the events bound by one RBP were cobound by the other (39.1\% to $60.2 \%)$ (Fig. 5F). In total, $17.1 \%$ of all the cassette exons regulated across heart development and $11.3 \%$ of those dysregulated in T1D hearts are cobound by CELF1 and RBFOX2 (Fig. 5F). Notably, most alternative splicing events that exhibit reciprocal regulation by the CELF and RBFOX families also change during heart development, myogenesis, and/or in T1D hearts (85.9\%) (Supplemental Fig. S6). Genes containing highly consistent, antagonistically coregulated events have previously been implicated in myogenesis and heart failure, such as Mef2d and Mef2a (Singh et al. 2014; Gao et al. 2016), and have numerous CELF and RBFOX binding sites proximal mammals, including chicken, fly, and worm (Ohno et al. 2012; Kuroyanagi et al. 2013; Blech-Hermoni et al. 2016; Conboy 2016). To examine if the splicing antagonism we observed in human and mouse was conserved more broadly across species, we used available RNA-seq data to identify cassette exons regulated by ancient CELF homologs and searched for the [U]GCAUG motif. Exons that were repressed by ancient CELF homologs in primary embryonic chicken cardiomyocytes (CELF1 depletion) (Fig. 6A), Drosophila indirect flight muscles (Bruno or Arrest depletion) (Fig. 6B), and C. elegans whole worm (unc-75 null mutant) (Fig. 6C) showed a striking enrichment of the RBFOX family [U]GCAUG motif downstream of regulated exons such that anywhere from $23.7 \%$ (worm) to $39.8 \%$ (chicken) contained this motif within 300 nt downstream. Importantly, genes containing these putative coregulated events in chicken, fly, and worm also show evidence of CELF/RBFOX coregulation within orthologous mammalian genes (Fig. 6D; Supplemental Table S7), suggesting that the functional consequences of this antagonism could be conserved. While the specific amino acid sequences affected by these putative coregulated events mostly differed between invertebrates and mammals, there are numerous examples of direct conservation of exons with characteristics of antagonistic coregulation between chicken and mammals (Supplemental Table S7). These results suggest that the antagonism between the CELF and RBFOX families we uncovered in human T cells (Figs. 1-4) and mouse muscle/heart (Fig. 5) displays ancient conservation throughout evolution.

\section{Discussion}

CELF2 and RBFOX2 are widely studied RNA binding proteins that both play critical roles in the regulation of tissue- and developmentally regulated splicing. In particular, both proteins have been studied extensively in neuromuscular tissues; however, cross-talk between these proteins has not been directly investigated. In studying the functional impact of CELF2 expression in Jurkat $\mathrm{T}$ cells, we find evidence for widespread antagonism of CELF2 and 

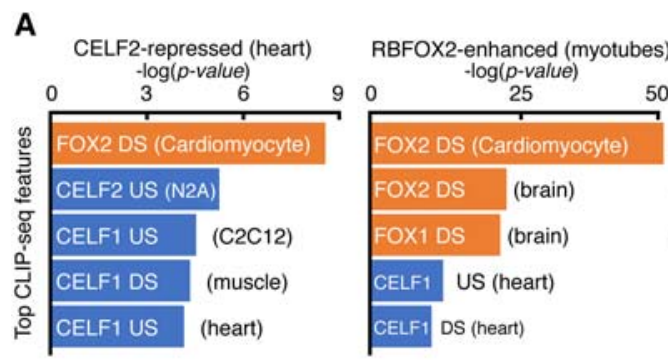

B 0.08 RBFOX2 CLIP peaks around CELF2 regulated exons

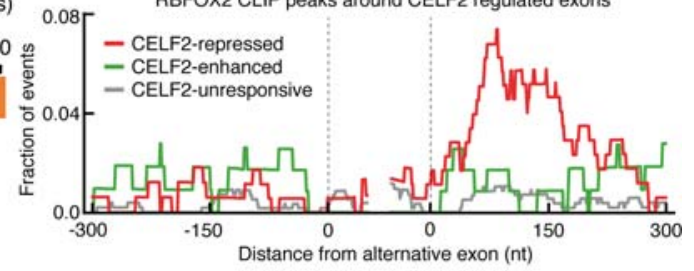

C Splicing events changing in: CELF heart OE Rbfox2 heart KO

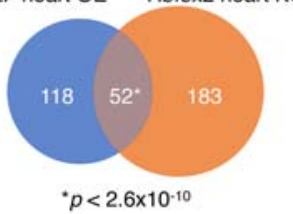

D

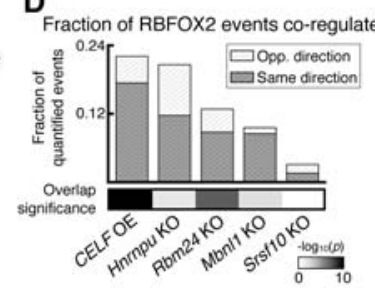

E

F

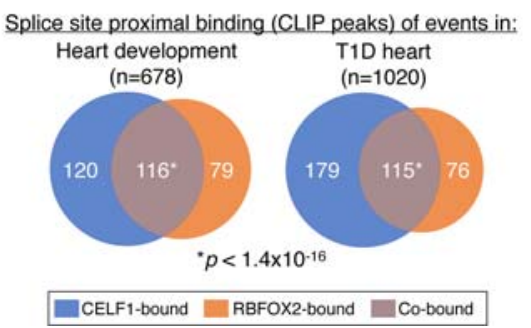

G

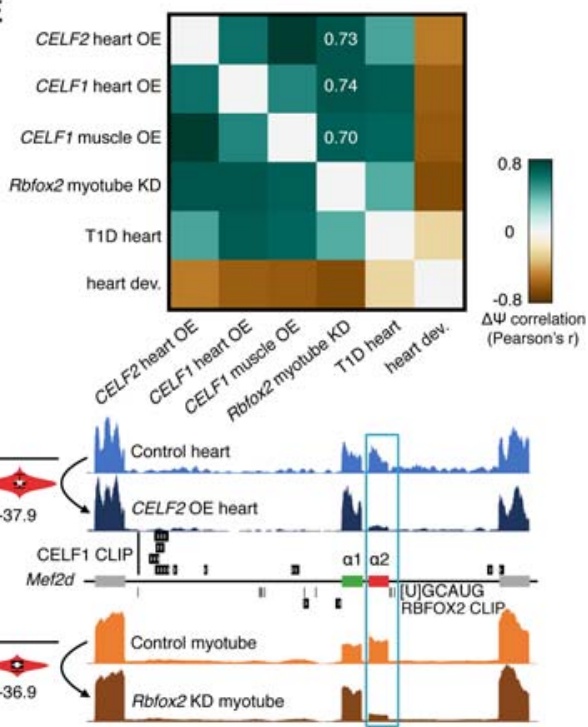

Figure 5. CELF/RBFOX antagonism is conserved in mouse heart/muscle and influences developmental and disease splicing programs. ( $A$ ) Two-tailed Fisher's exact test $-\log _{10}(P$-value) for the five most significant (lowest $P$-value) CLIP-seq peak occurrences enriched proximal to alternative exons repressed by CELF2 in mouse heart (left) or enhanced by RBFOX2 in myotubes (right) compared to unresponsive exons. Condition in which each CLIP experiment was carried out is indicated in parentheses. CLIP experiments for the CELF or RBFOX families are indicated in blue or orange, respectively. (DS) Downstream, (US) upstream. (B) RNA map of RBFOX2 CLIP-seq peaks from cardiomyocytes proximal to CELF2-repressed (red), -enhanced (green), or -unresponsive (gray) exons in the heart. (C) Venn diagram showing overlap of splicing changes in the mouse hearts that overexpressed (OE) CELF1 or CELF2 versus events altered through depletion of RBFOX2 (conditional heart KO) $\left(P<2.6 \times 10^{-10}\right.$, Fisher's exact test). (D) Fraction of total RBFOX2 regulated events that changed in the same direction (white) or the opposite direction (gray) of RBFOX2 regulation (top), with grayscale squares representing significance of the overlap between regulated events (bottom, $-\log _{10}[P$-value], Fisher's exact test). (E) Pairwise $E(\Delta \Psi)$ correlation for coregulated $(|E[\Delta \Psi]|>20 \%) L S V s$ between indicated conditions. (T1D) Type 1 diabetes hearts. (F) Venn diagrams showing number of cassette exons that contain a splice-site proximal CLIP-seq peak for CELF1 in heart (blue), RBFOX2 in cardiomyocytes (orange), or both (purple) and are regulated across heart development (left) or dysregulated in T1D hearts (right). (G) MAJIQ $\Delta \Psi$ violin plot (left) and UCSC Genome Browser view with RNA-seq tracks and CLIP-seq peaks (right) around a physiologically relevant event, the Mef2d $\alpha 1 / \alpha 2$ mutually exclusive exons, that is dysregulated in disease and antagonistically coregulated by CELF2 (top) and RBFOX2 (bottom) (for more examples, see Supplemental Table S3; Supplemental Figs. S6, S7).

RBFOX2 in regulating splicing (Figs. 1,2). Some of this antagonism is through binding of both CELF2 and RBFOX2 to individual target substrates, while for other substrates the reciprocal impact of CELF2 and RBFOX2 can be primarily attributed to the fact that CELF2 negatively regulates the expression of RBFOX2 (Figs. 3, 4). Importantly, we show that regulation of RBFOX 2 expression by CELF2 is not cell type-specific but rather also occurs in at least HEK293 fibroblasts. By analyzing publicly available data, we also find evidence for antagonism between CELF1 and CELF2 with RBFOX2 in heart and skeletal muscle in mice, as well as in chicken, Drosophila, and C. elegans (Figs. 5, 6). Therefore, we conclude that antagonism between CELF and RBFOX proteins is a conserved RBP network feature that is highly conserved throughout evolution.

\section{CELF2 represses expression of RBFOX2, while RBFOX2 counters the function of CELF2}

Antagonism between two RBPs can occur at either the level of function or expression. Functional antagonism often involves competitive binding in which binding of one RBP prevents the binding, and thus function, of the other, as seen in the PTB/ $\mathrm{nPTB}$ switch in the regulation of the N1 exon of Src (Markovtsov et al. 2000). Alternatively, two RBPs may bind simultaneously to a substrate and exert opposing influences on the spliceosome (Fu and Ares 2014). Antagonism at the level of expression also plays an important role in tuning RBP repertoire and activity, as transregulation of one RBP by others is common (Boutz et al. 2007; Huelga et al. 2012; Fu and Ares 2014).

We show here that antagonism between CELF2 and RBFOX2 involves both functional and expression antagonism. At least in Jurkat and HEK293 cells, CELF2 antagonizes expression of RBFOX2 protein through control of mRNA stability, as both the steady-state level and half-life of RBFOX2 mRNA are increased upon depletion of CELF2. Interestingly, we recently found that the stability of CELF2 mRNA is regulated by JNK signaling in activated T cells, which in turn is augmented by CELF2 (Martinez et al. 2015). Whether the stabilization of RBFOX2 and CELF2 mRNAs is mechanistically similar remains to be investigated; however, our

\section{Genome Research}

www.genome.org 
A
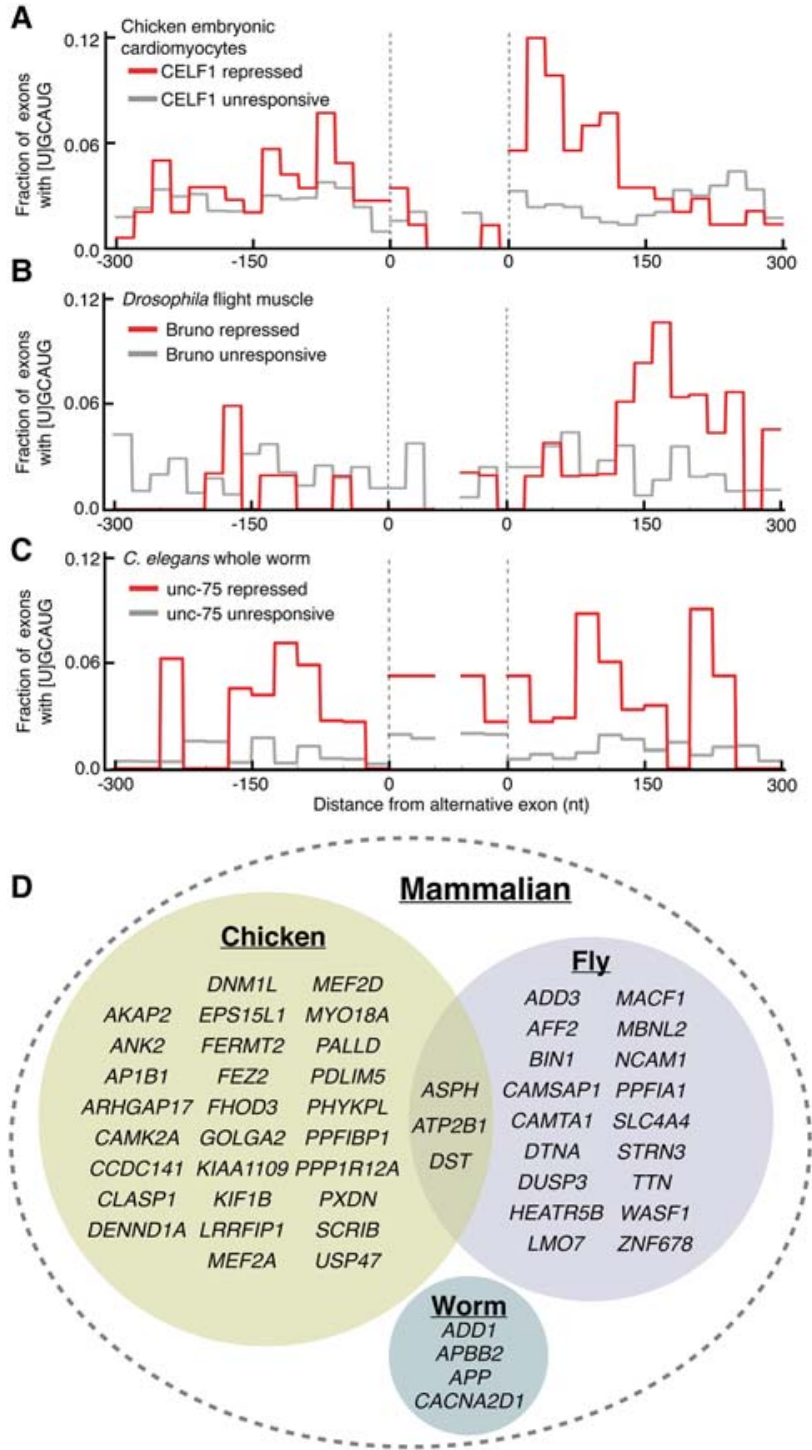

Figure 6. RBFOX motifs are enriched downstream of exons repressed by ancient CELF homologs. Histogram of the frequency of [U]GCAUG motifs in nonoverlapping 20-nt windows within 300 nt of alternative exons that are repressed by ancient CELF family homologs (red) or were unresponsive to depletion/mutation (gray) in $(A)$ chicken embryonic cardiomyocytes (control versus siRNA knockdown of CELF1) (Blech-Hermoni and Ladd 2015), (B) Drosophila melanogaster indirect flight muscles (control versus RNAi hairpin knockdown of bru1) (Spletter et al. 2015), and (C) C. elegans whole worm (control versus unc-75 null [e950] mutants) (Norris et al. 2014). (D) Venn diagram showing mammalian gene names containing CELF/RBFOX coregulated splicing where the orthologous gene in chicken, fly, or worm also contains a putative CELF/RBFOX coregulated event (see Methods and Supplemental Table S7).

data suggest that CELF2 protein may not be acting directly on the RBFOX2 mRNA. We also cannot fully rule out that additional layers of regulation do not also contribute to CELF2-mediated repression of RBFOX2 expression. Indeed, the fact that RBFOX2 levels do not change in stimulated Jurkat cells when CELF2 expression increases suggests either that the system is buffered to this threefold increase in CELF2 or that additional regulatory constraints of RBFOX2 expression exist. Importantly, antagonism of RBFOX2 expression by CELF 2 clearly has a broad impact on gene expression, as we identified at least 90 genes that lack any evidence for binding of CELF2 in the vicinity of the regulated exon and have clear evidence of RBFOX2 binding, for which direct overexpression of RBFOX2 (with no alteration in CELF2 expression) phenocopies the splicing effect observed upon depletion of CELF2. We also identified $\sim 40$ genes that are regulated by functional antagonism of CELF2/RBFOX2, as they have evidence for binding by both CELF2 and RBFOX2, exhibit distinct levels of exon inclusion in doubly deficient cells versus those depleted for either CELF2 or RBFOX2 alone, and depletion of CELF2 and RBFOX2 has opposing consequences.

Importantly, antagonism between RBFOX2 by CELF2 may help explain the bias in CELF2 versus RBFOX2 expression that has been observed in several tissues. At one extreme, CELF2 is highly expressed in thymus/immune tissues where the RBFOX proteins show minimal expression (Mallory et al. 2011, 2015; and this study), while at the other end of the spectrum, heart and skeletal muscle down-regulate CELF protein expression during development but express abundant levels of RBFOX proteins (Kalsotra et al. 2008; Singh et al. 2014). Notably, both CELF2 and RBFOX2 are highly expressed and nuclear-localized in the brain (Otsuka et al. 2009; Lee et al. 2016). While it is unclear how RBFOX2 avoids down-regulation by CELF2 in neurons, functional antagonism between CELF2 and RBFOX2 may help explain the subset of RBFOX targets that show differential splicing between muscle and brain (Zhang et al. 2008). A number of events examined here (e.g., $M A P 2 K 7, M E F 2 D, E P B 41$ ) are entirely consistent with this model in that they show high levels of exon inclusion in heart but lower in brain (MR Gazzara and Y Barash, unpubl.).

\section{Evidence for broader competition between CELF and RBFOX families}

In this study, we have focused on the interplay of CELF2 and RBFOX2 with regard to alternative splicing. However, we predict the antagonism we observe here extends to other CELF and RBFOX family members and other gene-regulatory mechanisms. Indeed, in Figure 5, we show that splicing changes induced by overexpression of CELF1 in muscle and heart correlate with RBFOX2 depletion, just as CELF2 overexpression correlates with RBFOX2 depletion. Moreover, recent work from the Black and Martin groups has revealed a role for cytoplasmic RBFOX1 in the brain in regulating transcript stability and/or translation (Lee et al. 2016). Notably, CELF4 is also highly expressed and localized to the cytoplasm in neurons (Wagnon et al. 2012). Analysis of iCLIP from mouse brain for CELF4 (Wagnon et al. 2012) shows nearly $90 \%$ of the cytoplasmic RBFOX1 binding sites within $3^{\prime}$ UTRs (Lee et al. 2016) are also bound by CELF4 (MR Gazzara and Y Barash, unpubl.). More generally, CELF proteins have been shown to recruit deadenylases to repress translation and destabilize mRNAs, whereas RBFOX promotes polyadenylation, stability, and translation (Wagnon et al. 2012; Lee et al. 2016). Therefore, interplay of CELF and RBFOX proteins may have a broad role in shaping gene expression at multiple stages of RNA processing throughout many tissues and developmental conditions.

\section{Cooperation and competition with other RBPs is a common feature of CELF and RBFOX families}

Antagonism between CELF2 and RBFOX2 is not the only example of functional interplay between these proteins and other RBPs. From the earliest studies linking CUG-repeat expansion to myotonic dystrophy, much work has focused on antagonism between the 
CELF and Muscleblind (MBNL) proteins (Pascual et al. 2006). CELF and MBNL proteins primarily exhibit functional antagonism in competing for binding to CUG/UG-repeats, although evidence for antagonistic expression exists as well (Wang et al. 2015). Interestingly, more recent work has shown cooperativity between MBNL and RBFOX proteins in iPSC reprogramming and muscle differentiation as well as in a myotonic dystrophy model (Venables et al. 2013; Klinck et al. 2014). RBFOX proteins have also been shown to regulate splicing in cooperation with other individual RBPs such as ESRP (Dittmar et al. 2012), PTBP (Li et al. 2015), and HNRNPH1 (Sun et al. 2011). In particular, recent work has demonstrated that RBFOX proteins in mammals often function through the activity of a large multiprotein assembly that influences the binding and activity of RBFOX (LASR) (Damianov et al. 2016). More broadly, antagonistic expression and activity among regulators of gene expression has long been understood to be central to many binary decisions in physiology, as it allows for sharp thresholds of activity (Davidson and Levine 2008). Indeed, antagonistic relationships between transcription factors have been shown to be critical to many cell fate decisions during development (Graf and Enver 2009) and have also been widely described in the activity of RBPs in regulating alternative splicing (Smith and Valcarcel 2000). In sum, the antagonistic relationship between CELF2 and RBFOX 2 that we describe here is only one of many positive and negative interactions between these and other RBPs that, together, shape the ultimate gene regulatory landscape of cells.

\section{Implications of CELF2/RBFOX2 antagonism for understanding human disease}

Finally, misregulation or misexpression of both CELF2 and RBFOX2 have been implicated as contributors to human disease including cancer, muscular dystrophies, heart failure, and Type 1 diabetes (Kuyumcu-Martinez et al. 2007; Cooper et al. 2009; Verma et al. 2013; Nutter et al. 2016). Our finding of mutual antagonism between these proteins suggests that disease-relevant splicing attributed previously to CELF2 or RBFOX2 may also be impacted by altered expression of the other. For example, Mef $2 d$ exon $\alpha 2$ is specifically included in heart and muscle and is known to be regulated by the RBFOX family (Singh et al. 2014). Inclusion of the $\alpha 2$ exon alters the phosphorylation and interaction partners of MEF2D to activate transcription of key myogenic genes during myogenesis (Sebastian et al. 2013). Importantly, previous studies have demonstrated that inclusion of Mef2d exon $\alpha 2$ is dysregulated in heart failure where RBFOX family members are down-regulated (Singh et al. 2014; Gao et al. 2016). Here, we show that splicing of Mef $2 d$ exon $\alpha 2$ is also dysregulated upon overexpression of CELF2 in heart (Fig. 5G) as well as overexpression of CELF1 in heart and muscle and in T1D (Supplemental Table S3; Supplemental Fig. S7). Other examples of developmental- and disease-relevant targets of both CELF proteins and RBFOX2 are summarized in Supplemental Table S3 and Supplemental Figure S6 and include genes involved in calcium signaling and other members of the MEF2 family of transcription factors. In all, these results suggest that antagonism between these two families of splicing factors contributes to the altered splicing patterns in physiologically relevant targets in developmental or disease contexts in which members of one or both families are altered. More broadly, our results emphasize the need to consider the interplay between RBPs when predicting or interpreting how dysregulation of one RBP may contribute to human disease and in designing potential therapeutic strategies.

\section{Methods}

\section{Cell culture, Western blots, and RT-PCR}

Growth, transfection, and stimulation of JSL1 Jurkat cells and HEK293 cells were done by standard methods. CELF2 and RBFOX2 were depleted by lentiviral shRNA expression of sequences: GCAGAGTAAAGGTTGTTGTTT (CELF2) and CCTTTAAATTT CTGCCTTTAAT (RBFOX2). Antibodies used for Western blots were as follows: CELF2 (University of Florida Hybridoma Lab, HL1889), RBFOX2, (Bethyl Labs, A300-864A), HNRNPL (Abcam, ab6106), and FLAG (Cell Signaling, \#2368). Low cycle RT-PCR was performed as described previously (Martinez et al. 2015) using primers specified in Supplemental Table S4.

\section{RNA-seq and analysis}

Total RNA was isolated, purified by DNase treatment and an RNeasy kit (Qiagen) and sent to the Iowa Institute of Human Genetics (https://www.medicine.uiowa.edu/humangenetics/dna/) for poly(A) selection, library preparation, and paired-end sequencing. Reads were processed by DESeq (Anders et al. 2010) and MAJIQ (Vaquero-Garcia et al. 2016) as described previously. Analysis of motifs and conservation is described in Supplemental Methods.

\section{CLIP-seq analysis}

eCLIP and iCLIP data were those available as of October 2016 through the ENCODE Portal (www.encodeproject.org) (Sundararaman et al. 2016). Additional publicly available CLIP-seq data sets in human and mouse (summarized with accession numbers in Supplemental Table S6) were also processed using the ENCODE eCLIP pipeline (Van Nostrand et al. 2016). High confidence RBFOX2 peaks are those that are significant by a two-tailed Fisher's exact test and contain the [U]GCAUG consensus motif within the boundary of the peak coordinates.

\section{Identification of ancient coregulated orthologs}

To identify putative CELF/RBFOX coregulated events across species, we defined cassette exons regulated by CELF family members in chicken, fly, and worm with $|\mathrm{E}(\Delta \Psi)| \geq 10 \%$, and scored for exons that also contained an RBFOX [U]GCAUG motif within or flanking the alternative exon. Mammalian orthologs for those genes containing event(s) that met these criteria were obtained using DIPOT (Hu et al. 2011). Analysis of conservation is further described in Supplemental Methods.

\section{Data access}

All sequencing data from this study have been submitted to the NCBI Gene Expression Omnibus (GEO; http://www.ncbi.nlm. nih.gov/geo/) under accession number GSE93594.

\section{Acknowledgments}

We thank all the members of the Barash and Lynch laboratories for helpful discussions and advice, particularly Caleb Radens and Jorge Vaquero-Garcia. We also thank the Genomics Division of the Iowa Institute of Human Genetics for their assistance with the RNA-seq. This work was funded by NIH grants from the National Institute on Aging, R01 AG046544 (Y.B.) and from the National Institute of General Medical Sciences, R35 GM118048 (K.W.L.).

\section{Genome Research}

www.genome.org 


\section{References}

Ajith S, Gazzara MR, Cole BS, Shankarling G, Martinez NM, Mallory MJ Lynch KW. 2016. Position-dependent activity of CELF2 in the regulation of splicing and implications for signal-responsive regulation in $\mathrm{T}$ cells. RNA Biol 13: 569-581.

Anders S, Huber W. 2010. Differential expression analysis for sequence count data. Genome Biol 11: R106.

Barash Y, Vaquero-Garcia J, Gonzalez-Vallinas J, Xiong HY, Gao W, Lee LJ, Frey BJ. 2013. AVISPA: a web tool for the prediction and analysis of alternative splicing. Genome Biol 14: R114.

Blech-Hermoni Y, Ladd AN. 2015. Identification of transcripts regulated by CUG-BP, Elav-like family member 1 (CELF1) in primary embryonic cardiomyocytes by RNA-seq. Genome Data 6: 74-76.

Blech-Hermoni Y, Dasgupta T, Coram RJ, Ladd AN. 2016. Identification of targets of CUG-BP, Elav-like family member 1 (CELF1) regulation in embryonic heart muscle. PLoS One 11: e0149061.

Boutz PL, Stoilov P, Li Q, Lin CH, Chawla G, Ostrow K, Shiue L, Ares M Jr, Black DL. 2007. A post-transcriptional regulatory switch in polypyrimidine tract-binding proteins reprograms alternative splicing in developing neurons. Genes Dev 21: 1636-1652.

Charlet BN, Logan P, Singh G, Cooper TA. 2002. Dynamic antagonism between ETR-3 and PTB regulates cell type-specific alternative splicing. Mol Cell 9: 649-658.

Conboy JG. 2016. Developmental regulation of RNA processing by Rbfox proteins. Wiley Interdiscip Rev RNA doi: 10.1002/wrna.1398.

Conway AE, Van Nostrand EL, Pratt GA, Aigner S, Wilbert ML, Sundararaman B, Freese P, Lambert NJ, Sathe S, Liang TY, et al. 2016. Enhanced CLIP uncovers IMP protein-RNA targets in human pluripotent stem cells important for cell adhesion and survival. Cell Rep 15: 666-679.

Cooper TA, Wan L, Dreyfuss G. 2009. RNA and disease. Cell 136: 777-793.

Damianov A, Black DL. 2010. Autoregulation of Fox protein expression to produce dominant negative splicing factors. RNA 16: 405-416.

Damianov A, Ying Y, Lin CH, Lee JA, Tran D, Vashisht AA, Bahrami-Samani E, Xing Y, Martin KC, Wohlschlegel JA, et al. 2016. Rbfox proteins regulate splicing as part of a large multiprotein complex LASR. Cell 165: 606-619.

Dasgupta T, Ladd AN. 2011. The importance of CELF control: molecular and biological roles of the CUG-BP, Elav-like family of RNA-binding proteins. Wiley Interdiscip Rev RNA 3: 104-121.

Davidson EH, Levine MS. 2008. Properties of developmental gene regulatory networks. Proc Natl Acad Sci 105: 20063-20066.

Dembowski JA, Grabowski PJ. 2009. The CUGBP2 splicing factor regulates an ensemble of branchpoints from perimeter binding sites with implications for autoregulation. PLoS Genet 5: e1000595.

Dittmar KA, Jiang P, Park JW, Amirikian K, Wan J, Shen S, Xing Y, Carstens RP. 2012. Genome-wide determination of a broad ESRP-regulated posttranscriptional network by high-throughput sequencing. Mol Cell Biol 32: $1468-1482$.

Fu XD, Ares M Jr. 2014. Context-dependent control of alternative splicing by RNA-binding proteins. Nat Rev Genet 15: 689-701.

Gallagher TL, Arribere JA, Geurts PA, Exner CR, McDonald KL, Dill KK, Marr HL, Adkar SS, Garnett AT, Amacher SL, et al. 2011. Rbfox-regulated alternative splicing is critical for zebrafish cardiac and skeletal muscle functions. Dev Biol 359: 251-261.

Gao C, Ren S, Lee JH, Qiu J, Chapski DJ, Rau CD, Zhou Y, Abdellatif M, Nakano A, Vondriska TM, et al. 2016. RBFox1-mediated RNA splicing regulates cardiac hypertrophy and heart failure. J Clin Invest 126: 195-206.

Gehman LT, Meera P, Stoilov P, Shiue L, O’Brien JE, Meisler MH, Ares M Jr, Otis TS, Black DL. 2012. The splicing regulator Rbfox 2 is required for both cerebellar development and mature motor function. Genes Dev 26: $445-460$

Giudice J, Xia Z, Wang ET, Scavuzzo MA, Ward AJ, Kalsotra A, Wang W, Wehrens $\mathrm{XH}$, Burge $\mathrm{CB}$, Li W, et al. 2014. Alternative splicing regulates vesicular trafficking genes in cardiomyocytes during postnatal heart development. Nat Commun 5: 3603.

Graf T, Enver T. 2009. Forcing cells to change lineages. Nature 462: 587-594.

Han J, Cooper TA. 2005. Identification of CELF splicing activation and repression domains in vivo. Nucleic Acids Res 33: 2769-2780.

$\mathrm{Hu}$ Y, Flockhart I, Vinayagam A, Bergwitz C, Berger B, Perrimon N, Mohr SE. 2011. An integrative approach to ortholog prediction for disease-focused and other functional studies. BMC Bioinformatics 12: 357.

Huelga SC, Vu AQ, Arnold JD, Liang TY, Liu PP, Yan BY, Donohue JP, Shiue L, Hoon S, Brenner S, et al. 2012. Integrative genome-wide analysis reveals cooperative regulation of alternative splicing by hnRNP proteins. Cell Rep 1: 167-178.

Kalsotra A, Cooper TA. 2011. Functional consequences of developmentally regulated alternative splicing. Nat Rev Genet 12: 715-729.
Kalsotra A, Xiao X, Ward AJ, Castle JC, Johnson JM, Burge CB, Cooper TA. 2008. A postnatal switch of CELF and MBNL proteins reprograms alternative splicing in the developing heart. Proc Natl Acad Sci 105: 20333-20338.

Kalsotra A, Wang K, Li PF, Cooper TA. 2010. MicroRNAs coordinate an alternative splicing network during mouse postnatal heart development. Genes Dev 24: 653-658.

Klinck R, Fourrier A, Thibault P, Toutant J, Durand M, Lapointe E, CailletBoudin ML, Sergeant N, Gourdon G, Meola G, et al. 2014. RBFOX1 cooperates with MBNL1 to control splicing in muscle, including events altered in myotonic dystrophy type 1. PLoS One 9: e107324.

Kuroyanagi H, Ohno G, Mitani S, Hagiwara M. 2007. The Fox-1 family and SUP-12 coordinately regulate tissue-specific alternative splicing in vivo. Mol Cell Biol 27: 8612-8621.

Kuroyanagi H, Watanabe Y, Suzuki Y, Hagiwara M. 2013. Position-dependent and neuron-specific splicing regulation by the CELF family RNAbinding protein UNC-75 in Caenorhabditis elegans. Nucleic Acids Res 41: $4015-4025$.

Kuyumcu-Martinez NM, Wang GS, Cooper TA. 2007. Increased steady-state levels of CUGBP1 in myotonic dystrophy 1 are due to PKC-mediated hyperphosphorylation. Mol Cell 28: 68-78.

Ladd AN, Taffet G, Hartley C, Kearney DL, Cooper TA. 2005. Cardiac tissuespecific repression of CELF activity disrupts alternative splicing and causes cardiomyopathy. Mol Cell Biol 25: 6267-6278.

Lambert N, Robertson A, Jangi M, McGeary S, Sharp PA, Burge CB. 2014. RNA Bind-n-Seq: quantitative assessment of the sequence and structural binding specificity of RNA binding proteins. Mol Cell 54: 887-900.

Lee JA, Damianov A, Lin CH, Fontes M, Parikshak NN, Anderson ES Geschwind DH, Black DL, Martin KC. 2016. Cytoplasmic Rbfox1 regulates the expression of synaptic and autism-related genes. Neuron 89: 113-128.

Li H, Qiu J, Fu XD. 2012. RASL-seq for massively parallel and quantitative analysis of gene expression. Curr Protoc Mol Biol Chapter 4: Unit 4.13: 1-9.

Li YI, Sanchez-Pulido L, Haerty W, Ponting CP. 2015. RBFOX and PTBP1 proteins regulate the alternative splicing of micro-exons in human brain transcripts. Genome Res 25: 1-13.

Mallory MJ, Jackson J, Weber B, Chi A, Heyd F, Lynch KW. 2011. Signal- and development-dependent alternative splicing of LEF1 in T cells is controlled by CELF2. Mol Cell Biol 31: 2184-2195.

Mallory MJ, Allon SJ, Qiu J, Gazzara MR, Tapescu I, Martinez NM, Fu XD, Lynch KW. 2015. Induced transcription and stability of CELF2 mRNA drives widespread alternative splicing during T-cell signaling. Proc Natl Acad Sci 112: E2139-E2148.

Markovtsov V, Nikolic JM, Goldman JA, Turck CW, Chou MY, Black DL. 2000. Cooperative assembly of an hnRNP complex induced by a tissue-specific homolog of polypyrimidine tract binding protein. Mol Cell Biol 20: 7463-7479.

Martinez NM, Agosto L, Qiu J, Mallory MJ, Gazzara MR, Barash Y, Fu XD, Lynch KW. 2015. Widespread JNK-dependent alternative splicing induces a positive feedback loop through CELF2-mediated regulation of MKK7 during T-cell activation. Genes Dev 29: 2054-2066.

Norris AD, Gao S, Norris ML, Ray D, Ramani AK, Fraser AG, Morris Q Hughes TR, Zhen M, Calarco JA. 2014. A pair of RNA-binding proteins controls networks of splicing events contributing to specialization of neural cell types. Mol Cell 54: 946-959.

Nutter CA, Jaworski EA, Verma SK, Deshmukh V, Wang Q, Botvinnik OB, Lozano MJ, Abass IJ, Ijaz T, Brasier AR, et al. 2016. Dysregulation of RBFOX2 is an early event in cardiac pathogenesis of diabetes. Cell Rep 15: $2200-2213$.

Ohno G, Ono K, Togo M, Watanabe Y, Ono S, Hagiwara M, Kuroyanagi H. 2012. Muscle-specific splicing factors ASD-2 and SUP-12 cooperatively switch alternative pre-mRNA processing patterns of the ADF/cofilin gene in Caenorhabditis elegans. PLoS Genet 8: e1002991.

Otsuka N, Tsuritani K, Sakurai T, Kato K, Matoba R, Itoh J, Okuyama S, Yamada K, Yoneda Y. 2009. Transcriptional induction and translational inhibition of Arc and Cugbp2 in mice hippocampus after transient global ischemia under normothermic condition. Brain Res 1287: 136-145.

Pascual M, Vicente M, Monferrer L, Artero R. 2006. The Muscleblind family of proteins: an emerging class of regulators of developmentally programmed alternative splicing. Differentiation 74: 65-80.

Pedrotti S, Giudice J, Dagnino-Acosta A, Knoblauch M, Singh RK, Hanna A, Mo Q, Hicks J, Hamilton S, Cooper TA. 2015. The RNA-binding protein Rbfox1 regulates splicing required for skeletal muscle structure and function. Hum Mol Genet 24: 2360-2374.

Ponthier JL, Schluepen C, Chen W, Lersch RA, Gee SL, Hou VC, Lo AJ, Short SA, Chasis JA, Winkelmann JC, et al. 2006. Fox-2 splicing factor binds to a conserved intron motif to promote inclusion of protein 4.1R alternative exon 16. J Biol Chem 281: 12468-12474.

Sebastian S, Faralli H, Yao Z, Rakopoulos P, Palii C, Cao Y, Singh K, Liu QC, Chu A, Aziz A, et al. 2013. Tissue-specific splicing of a ubiquitously 
expressed transcription factor is essential for muscle differentiation. Genes Dev 27: 1247-1259.

Singh RK, Xia Z, Bland CS, Kalsotra A, Scavuzzo MA, Curk T, Ule J, Li W, Cooper TA. 2014. Rbfox2-coordinated alternative splicing of Mef2d and Rock 2 controls myoblast fusion during myogenesis. Mol Cell 55: $592-603$.

Smith CWJ, Valcarcel J. 2000. Alternative pre-mRNA splicing: the logic of combinatorial control. Trends Biochem Sci 25: $381-388$.

Spletter ML, Barz C, Yeroslaviz A, Schonbauer C, Ferreira IR, Sarov M, Gerlach D, Stark A, Habermann BH, Schnorrer F. 2015. The RNA-binding protein Arrest (Bruno) regulates alternative splicing to enable myofibril maturation in Drosophila flight muscle. EMBO Rep 16: 178-191.

Sun S, Zhang Z, Fregoso O, Krainer AR. 2011. Mechanisms of activation and repression by the alternative splicing factors RBFOX1/2. RNA 18: $274-283$.

Sundararaman B, Zhan L, Blue SM, Stanton R, Elkins K, Olson S, Wei X, Van Nostrand EL, Pratt GA, Huelga SC, et al. 2016. Resources for the comprehensive discovery of functional RNA elements. Mol Cell 61: 903-913.

Taliaferro JM, Lambert NJ, Sudmant PH, Dominguez D, Merkin JJ, Alexis MS, Bazile CA, Burge CB. 2016. RNA sequence context effects measured in vitro predict in vivo protein binding and regulation. Mol Cell 64: 294-306.

Underwood JG, Boutz PL, Dougherty JD, Stoilov P, Black DL. 2005. Homologues of the Caenorhabditis elegans Fox-1 protein are neuronal splicing regulators in mammals. Mol Cell Biol 25: 10005-10016.

Van Nostrand EL, Pratt GA, Shishkin AA, Gelboin-Burkhart C, Fang MY, Sundararaman B, Blue SM, Nguyen TB, Surka C, Elkins K, et al. 2016. Robust transcriptome-wide discovery of RNA-binding protein binding sites with enhanced CLIP (eCLIP). Nat Methods 13: 508-514.

Vaquero-Garcia J, Barrera A, Gazzara MR, Gonzalez-Vallinas J, Lahens NF, Hogenesch JB, Lynch KW, Barash Y. 2016. A new view of transcriptome complexity and regulation through the lens of local splicing variations. eLife 5: e11752.
Venables JP, Lapasset L, Gadea G, Fort P, Klinck R, Irimia M, Vignal E, Thibault P, Prinos P, Chabot B, et al. 2013. MBNL1 and RBFOX2 cooperate to establish a splicing programme involved in pluripotent stem cell differentiation. Nat Commun 4: 2480.

Verma SK, Deshmukh V, Liu P, Nutter CA, Espejo R, Hung ML, Wang GS, Yeo GW, Kuyumcu-Martinez MN. 2013. Reactivation of fetal splicing programs in diabetic hearts is mediated by protein kinase $\mathrm{C}$ signaling. J Biol Chem 288: 35372-35386.

Wagnon JL, Briese M, Sun W, Mahaffey CL, Curk T, Rot G, Ule J, Frankel WN. 2012. CELF4 regulates translation and local abundance of a vast set of mRNAs, including genes associated with regulation of synaptic function. PLoS Genet 8: e1003067.

Wang ET, Ward AJ, Cherone JM, Giudice J, Wang TT, Treacy DJ, Lambert NJ, Freese P, Saxena T, Cooper TA, et al. 2015. Antagonistic regulation of mRNA expression and splicing by CELF and MBNL proteins. Genome Res 25: 858-871.

Wei C, Qiu J, Zhou Y, Xue Y, Hu J, Ouyang K, Baneriee I, Zhang C, Chen B, Li $\mathrm{H}$, et al. 2015. Repression of the central splicing regulator RBFox2 is functionally linked to pressure overload-induced heart failure. Cell Rep 10: $1521-1533$

Yeo GW, Coufal NG, Liang TY, Peng GE, Fu XD, Gage FH. 2009. An RNA code for the FOX2 splicing regulator revealed by mapping RNA-protein interactions in stem cells. Nat Struct Mol Biol 16: 130-137.

Zhang W, Liu H, Han K, Grabowski PJ. 2002. Region-specific alternative splicing in the nervous system: implications for regulation by the RNA-binding protein NAPOR. RNA 8: 671-685.

Zhang C, Zhang Z, Castle J, Sun S, Johnson J, Krainer AR, Zhang MQ. 2008. Defining the regulatory network of the tissue-specific splicing factors Fox-1 and Fox-2. Genes Dev 22: 2550-2563.

Received January 11, 2017; accepted in revised form May 8, 2017.

\section{Genome Research}




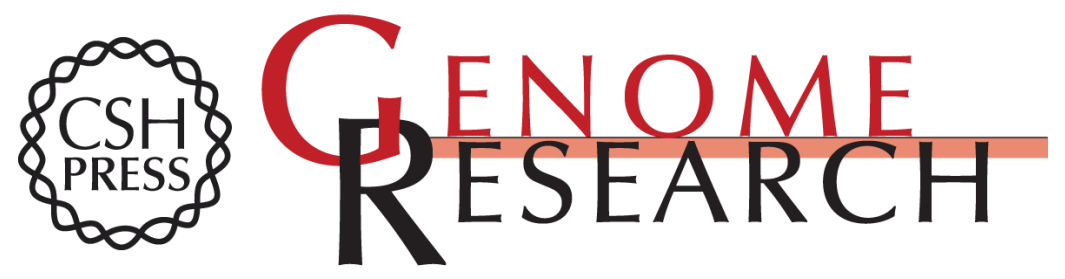

\section{Ancient antagonism between CELF and RBFOX families tunes mRNA splicing outcomes}

Matthew R. Gazzara, Michael J. Mallory, Renat Roytenberg, et al.

Genome Res. 2017 27: 1360-1370 originally published online May 16, 2017 Access the most recent version at doi:10.1101/gr.220517.117

Supplemental Material

References

Creative Commons

License

Email Alerting Service
http://genome.cshlp.org/content/suppl/2017/06/21/gr.220517.117.DC1

This article cites 65 articles, 21 of which can be accessed free at: http://genome.cshlp.org/content/27/8/1360.full.html\#ref-list-1

This article is distributed exclusively by Cold Spring Harbor Laboratory Press for the first six months after the full-issue publication date (see

http://genome.cshlp.org/site/misc/terms.xhtml). After six months, it is available under a Creative Commons License (Attribution-NonCommercial 4.0 International), as described at http://creativecommons.org/licenses/by-nc/4.0/.

Receive free email alerts when new articles cite this article - sign up in the box at the top right corner of the article or click here.

\section{Affordable, Accurate Sequencing.}

